\title{
THE EINSTEIN-WEYL EQUATIONS, SCATTERING MAPS, AND HOLOMORPHIC DISKS
}

\author{
Claude LeBrun and L.J. Mason
}

\begin{abstract}
We show that conformally compact, space-time oriented, globally hyperbolic, Lorentzian Einstein-Weyl 3-manifolds are in natural one-to-one correspondence with orientation-reversing diffeomorphisms of the complex projective line. The proof hinges on a holomorphic-disk analog of Hitchin's mini-twistor correspondence.
\end{abstract}

Recent years have witnessed a resurgence of twistor methods in both mathematics and physics. On the physics side, this has primarily been driven by Witten's discovery [21] that strings in twistor space can be used to calculate Yang-Mills scattering amplitudes, resulting in concrete experimental predictions that can be tested using existing particle accelerators. One of the main strands of thought here [1] involves the introduction of open strings — Riemann surfaces with non-empty boundary, where the boundary is constrained to lie on some specified submanifold, called a $D$-brane.

On the mathematical side, there has been a parallel development. Penrose-type twistor correspondences study moduli spaces of compact holomorphic curves $C$ in a complex manifold $Z$; when $Z$ is equipped with an anti-holomophic involution $\sigma: Z \rightarrow Z$, the parameter space for those curves $C \subset Z$ in a fixed homology class with $\sigma(C)=C$ typically turns out to carry a natural geometrical structure, and often describes the general real-analytic solution of some interesting differential-geometric problem. By contrast, the new paradigm is instead to study the moduli spaces of holomorphic curves-with-boundary $C$, where $\partial C \neq \varnothing$ is constrained to lie in a totally real submanifold $P \subset Z$. It turns out that this framework is well adapted to the study of many natural problems in global differential geometry where solutions typically are of very low regularity. For example, Zoll metrics (and, more generally, Zoll projective structures) on surfaces turn out to arise [14] from holomorphic disks in $\mathbb{C P}_{2}$ with boundaries in a totally real $\mathbb{R P}^{2} \hookrightarrow \mathbb{C P}_{2}$. Analogous results [15] describe split-signature self-dual conformal structures on 4-manifolds in terms of holomorphic disks in $\mathbb{C P}_{3}$ with boundary on a totally real $\mathbb{R P}^{3} \hookrightarrow \mathbb{C P}_{3}$. For closely-related results on the Yang-Mills equations in split signature, see [16].

This article will show that these techniques also lead to definitive results concerning the Einstein-Weyl equations for a 3-dimensional Lorentzian space-time, thereby substantiating a claim made in [13]. Recall that the Einstein-Weyl equations, for a conformal class of metrics $[g]$ and a compatible torson-free connection $\nabla$, are precisely the requirement that the trace-free symmetric part of the Ricci tensor of $\nabla$ should

Received by the editors July 21, 2008.

Supported in part by NSF grant DMS-0604735.

Supported in part by the EU through FP6 Marie Curie RTN ENIGMA (MRTN-CT-2004-5652). 
vanish; that is, the torsion-free connection $\nabla$ is required to satisfy $\nabla g=\alpha \otimes g$ for some 1 -form $\alpha$, and its curvature tensor $\mathcal{R}$ is required to satisfy

$$
\mathcal{R}^{k}{ }_{i k j}+\mathcal{R}^{k}{ }_{j k i}=f g_{i j}
$$

for some function $f$. In three dimensions, these equations were first investigated by Elie Cartan [3], who discovered (in essence) that they are totally integrable. Much later, Hitchin [8] rediscovered the 3-dimensional Einstein-Weyl equations as a dimensional reduction of the 4-dimensional self-duality equations, and described their Riemannian solutions in terms of the so-called mini-twistor correspondence $[9,10]$. The twistor correspondence we will develop here is a close cousin of Hitchin's mini-twistor correspondence, but is naturally adapted to low-regularity Lorentzian solutions with very specific asymptotic behavior. Our main result is the following:

Theorem A. There is a natural one-to-one correspondence between

- smooth, space-time-oriented, conformally compact, globally hyperbolic, Lorentzian Einstein-Weyl 3-manifolds $(M,[g], \nabla)$; and

- orientation-reversing diffeomorphisms $\psi: \mathbb{C P}_{1} \rightarrow \mathbb{C P}_{1}$.

The prototypical example of such an Einstein-Weyl manifold is three-dimensional de Sitter space $S L(2, \mathbb{C}) / S L(2, \mathbb{R})$; and the diffeomorphism $\mathbb{C P}_{1} \rightarrow \mathbb{C P}_{1}$ corresponding to this prototype is the antipodal map

$$
\left[z_{0}: z_{1}\right] \longmapsto\left[-\bar{z}_{1}: \bar{z}_{0}\right] \text {. }
$$

In the present context, two Einstein-Weyl structures are to be considered identical if they are related by a connection-preserving conformal isometry. On the other hand, two orientation-reversing diffeomorphisms $\psi_{1}, \psi_{2}: \mathbb{C P}_{1} \rightarrow \mathbb{C P}_{1}$ are considered the same iff

$$
\psi_{1}=\varphi \circ \psi_{2} \circ \phi^{-1}
$$

for Möbius transformations $\varphi, \phi \in P S L(2, \mathbb{C})$ of the domain and range.

In one direction, a direct geometrical interpretation of this correspondence can be described in terms of scattering maps. We will thus begin by explaining how to associate such a map $\psi: \mathbb{C P}_{1} \rightarrow \mathbb{C P}_{1}$ to any Einstein-Weyl 3 -manifold $(M,[g], \nabla)$ satisfying the stated hypotheses.

Recall that a conformal Lorentzian $n$-manifold $(M,[g])$ is said to be space-time oriented if the structure group of its tangent bundle has been reduced to the identity component $S O^{\uparrow}(1, n-1) \times \mathbb{R}^{+}$of the conformal Lorentz group; in particular, this equips $M$ with a time orientation, allowing one to determine whether a time-like vector is past- or future-pointing. A time-oriented Lorentz manifold $(M,[g])$ is called globally hyperbolic if it contains a Cauchy surface, meaning a space-like hypersurface $\Sigma$ which meets every endless time-like or null curve exactly once; when this happens, there is then a diffeomorphism $M \approx \Sigma \times \mathbb{R}$ such that every level set $\Sigma \times\{t\}$ is a Cauchy surface $[5,20]$. We generalize the usual notion of conformal compactness for Einstein manifolds $[7,19]$ as in $[12]$, declaring that an Einstein-Weyl space $(M,[g], \nabla)$ is conformally compact iff, for some smooth compact pseudo-Riemannian manifoldwith-boundary $(X, \hat{g})$,

- there is a diffeomorphism $\Phi: M \stackrel{\approx}{\longrightarrow} X-\partial X$ with $\left[\Phi^{*} \hat{g}\right]=[g]$;

- the induced boundary metric $\left.\hat{g}\right|_{\partial X}$ is everywhere non-degenerate; and 
- if $\alpha$ is the 1 -form on $X-\partial X$ defined by $\nabla \hat{g}=\alpha \otimes \hat{g}$, then there is some non-degenerate defining function $u: X \rightarrow \mathbb{R}$ for $\partial X$ such that $\alpha-2 u^{-1} d u$ extends by zero to $X$ as a smooth 1 -form which vanishes along $\partial X$.

If $(M,[g])$ is globally hyperbolic, then $\partial X \subset X$ is automatically space-like, and $X \approx$ $\Sigma \times[0,1]$ for some compact $(n-1)$-manifold $\Sigma$. If $n=3$ and $M$ is space-time oriented, it then follows that $M$ is diffeomorphic to $\Sigma \times \mathbb{R}$ for some compact oriented surface $\Sigma$, and can be conformally compactified by adding two copies $\mathscr{I}_{-}$and $\mathscr{I}_{+}$of $\Sigma$, henceforth called past and future infinity.

Lemma 1. For an Einstein-Weyl manifold as above, let $p \in \mathscr{I}_{-}$be any point of past infinity. Then all the null geodesics emanating from $p$ refocus at a unique point $q \in \mathscr{I}_{+}$. Moreover, $\mathscr{I}_{-}$and $\mathscr{I}_{+}$are necessarily diffeomorphic to $S^{2}$.

Proof. First notice that every null geodesic in $(M,[g])$ can be extended to a null geodesic in $(X,[\hat{g}])$, and therefore has unique past and future end-points on $\mathscr{I}_{-}$and $\mathscr{I}_{+}$, respectively. Indeed, the null geodesics meeting $\mathscr{I}_{ \pm}$can be parameterized by the unit tangent bundle $U T \mathscr{I}_{ \pm}$, and following these backwards or forwards to a Cauchy surface $\Sigma \subset M$ then gives a local diffeomorphism $U T \mathscr{I}_{ \pm} \rightarrow U T \Sigma$ which must be onto, since the image is both open and compact. Using the conformal invariance of null geodesics, together with the fact that they are also geodesics of the Weyl connection $\nabla$, the hypothesized form of the singularity of $\nabla$ at $\partial X$ thus implies that the affine parameter $t$ on any inextendible null $\nabla$-geodesic must range over all of $\mathbb{R}$, and must approach $-\infty$ (respectively, $+\infty$ ) exactly when the geodesic approaches its ideal past (respectively, future) end-point $p \in \mathscr{I}_{-}$(respectively, $q \in \mathscr{I}_{+}$).

This said, let $\gamma: \mathbb{R} \rightarrow M$ now be a $\nabla$-affinely parameterized null geodesic in $(M,[g])$. Let $\mathbf{w}$ be a $\nabla$-parallel non-zero space-like vector field along $\gamma$ which is orthogonal to the null vector field $\mathbf{v}:=\gamma^{\prime}$. Now observe that, because $\nabla$ preserves the conformal structure,

$$
\mathcal{R} \in \Lambda^{2} \otimes\left(\Lambda^{2} \oplus \mathbb{R} g\right)
$$

after index lowering. Hence (1) implies that $\mathcal{R}_{\mathbf{v w}} \mathbf{v}$ is $g$-orthogonal to both $\mathbf{v}$ and $\mathbf{w}$, and is therefore a multiple of $\mathbf{v}$. It follows that there is a $\nabla$-Jacobi field $\tilde{\mathbf{w}}$ along $\gamma$ with $\tilde{\mathbf{w}} \equiv \mathbf{w} \bmod \mathbf{v}$. Thus $\mathbf{w} \bmod \mathbf{v}$ actually represents an infinitesimal variation of $\gamma$ through (unparameterized) null geodesics. However, we have assumed that $\nabla \hat{g}=\alpha \otimes \hat{g}$ where $\alpha-2 u^{-1} d u$ vanishes along $\mathscr{I}_{ \pm}$, so $t \sim \pm \log u$ as $\gamma$ approaches $\partial X$, and $\hat{g}(\mathbf{w}, \mathbf{w}) \rightarrow 0$ as our affine parameter $t \rightarrow \pm \infty$. Hence $\mathbf{w}$ actually represents an infinitesimal variation of $\gamma$ through null geodesics emanating from $p \in \mathscr{I}_{-}$and terminating at $q \in \mathscr{I}_{+}$. It follows that an infinitesimal change of the initial direction of a null geodesic emanating from $p \in \mathscr{I}_{-}$results in no change at all of the terminal end-point $q \in \mathscr{I}_{+}$. Since the set of future-pointing null directions at $p$ forms a connected family $\left(\approx S^{1}\right)$, we thus conclude that all of the null geodesics emanating from $p \in \mathscr{I}_{-}$terminate at the same point $q \in \mathscr{I}_{+}$.

The family of null geodesics emanating from an arbitrary point $p \in \mathscr{I}_{-}$and refocusing at some $q \in \mathscr{I}_{+}$now gives us an immersion of $\mathbb{R} \times S^{1}$ in $M$, since the corresponding Jacobi fields $\tilde{\mathbf{w}}$ are everywhere non-zero; moreover, its tangent space is null at every point. The closure of this surface in $X$, obtained by adding $p$ and $q$, is therefore a topological immersion of $S^{2}$. By truncating and capping off with space-like disks slightly to the future of $p$ and slightly to the past of $q \in X$, we may 
then smooth this object out into a differentiably immersed 2-sphere $\mathcal{S} \rightarrow M$ such that $T \mathcal{S}$ is then everwhere space-like or null. Following a time-like vector field then gives us an immersion $\varpi: \mathcal{S} \rightarrow \Sigma$ onto a Cauchy surface, since $T \mathcal{S}$ is everywhere nontimelike. Thus, $\varpi$ is necessarily a covering map; and since $\mathcal{S}$ is simply connected, this must actually be the universal cover of $\Sigma$. But since $\Sigma$ is an oriented surface, the deck transformations of $\varpi$ must be orientation-preserving. Since any orientation-preserving diffeomorphism of $S^{2}$ has a fixed point, it therefore follows that there cannot be any non-trivial deck transformations of $\varpi$, and $\varpi$ must therefore be a diffeomorphism. Hence $\Sigma$ and $\mathscr{I}_{ \pm} \approx \Sigma$ are 2 -spheres, as claimed.

Since the 2 -spheres $\mathscr{I}_{ \pm}$inherit an induced conformal structure from $X$, and since both are oriented by the space-orientation of $M$, each is isomorphic to $\mathbb{C P}_{1}$ in a manner unique up to a Möbius transformation. We may thus define the scattering map $\psi: \mathbb{C P}_{1} \rightarrow \mathbb{C P}_{1}$ associated with $(M,[g], \nabla)$ to be the function corresponding to

$$
\mathscr{I}_{-} \ni p \longmapsto q \in \mathscr{I}_{+}
$$

via some arbitrary choice of oriented conformal isomorphisms $\mathscr{I}_{ \pm} \cong \mathbb{C P}_{1}$. Since the scattering $p \mapsto q$ can locally be realized by following a congruence of null geodesics from $\mathscr{I}_{-}$to $\mathscr{I}_{+}, \psi$ is automatically smooth; and since we may construct a smooth inverse for it by instead following null geodesics backwards from $\mathscr{I}_{+}$to $\mathscr{I}_{-}$, we see that $\psi$ is in fact always a diffeomorphism.

The fact that $\psi$ is necessarily orientation-reversing results from $q \in \mathscr{I}_{+}$being the first point null-conjugate to $p \in \mathscr{I}_{-}$along any null geodesic $\gamma$ joining them. Indeed, letting $\mathbf{v}$ and $\mathbf{w}$ be as in the proof of Lemma 1 above, and letting $g$ be a choice of $g \in[g]$ which is parallel along $\gamma$, there are $\nabla$-Jacobi fields $\mathbf{w}_{1}$ and $\mathbf{w}_{2}$ along $\gamma$ such that $\mathbf{w}_{1} \equiv t \mathbf{w} \bmod \mathbf{v}$ and $g\left(\mathbf{w}_{2}, \mathbf{v}\right)=1$. The corresponding sections $\tilde{\mathbf{w}}_{1}$ and $\tilde{\mathbf{w}}_{2}$ of the normal bundle of $\gamma$ then join infinitesimally separated null geodesics, and so, by conformal invariance, extend to $X$. The determinant of $T_{p} \mathscr{I}_{-} \rightarrow T_{q} \mathscr{I}_{+}$is now explicitly given by

$$
\left.\left.\left(\tilde{\mathbf{w}}_{1} \wedge \tilde{\mathbf{w}}_{2}\right)\right|_{p} \longmapsto\left(\tilde{\mathbf{w}}_{1} \wedge \tilde{\mathbf{w}}_{2}\right)\right|_{q},
$$

and since $\tilde{\mathbf{w}}_{1} \wedge \tilde{\mathbf{w}}_{2}$ changes sign exactly once, at $t=0$, it thus follows that the Jacobian determinant of the scattering map is negative at $p$. The constructed map $\psi$ is therefore an orientation-reversing diffeomorphism, as promised.

We now turn to the problem of inverting the correspondence; and it is precisely here that twistor ideas will finally come to the fore. The graph of any orientationreversing diffeomorphism $\psi: \mathbb{C P}_{1} \rightarrow \mathbb{C P}_{1}$ is a totally real 2 -sphere $P \subset \mathbb{C P}_{1} \times \mathbb{C P}_{1}$. Our program is then to construct the corresponding 3-manifold $M=M_{\psi}$ as a family of holomorphic disks in $Z=\mathbb{C P}_{1} \times \mathbb{C P}_{1}$ with boundaries on $P \subset Z$. When $\psi$ is the antipodal map (2), these disks are explicitly given by

$$
\zeta \longmapsto([a \zeta+b: c \zeta+d],[-\bar{d} \zeta-\bar{c}: \bar{b} \zeta+\bar{a}])
$$

as $\zeta$ ranges over the unit disk $|\zeta| \leq 1$ in $\mathbb{C}$. Here, of course, each

$$
\left[\begin{array}{ll}
a & b \\
c & d
\end{array}\right] \in S L(2, \mathbb{C})
$$


represents a particular choice of parameterized disk. Notice that the the boundaries of these disks are exactly the standard round circles in $P \cong S^{2}$, and that the moduli space $M$ of disks modulo reparameterizations is exactly de Sitter space $S L(2, \mathbb{C}) / S L(2, \mathbb{R})$.

If we form the double of any of the above disks, its abstract normal bundle, in the sense of [13], will be $\mathcal{O}(2)$. Since $h^{1}\left(\mathbb{C P}_{1}, \mathcal{O}(2)\right)=0$ and $h^{0}\left(\mathbb{C P}_{1}, \mathcal{O}(2)\right)=3$, it follows that that they are all Fredholm regular, that the 3-parameter family which they form is locally complete, and that the family is stable under totally real deformations of $P$; cf. McDuff-Salamon [17, Appendix C] for a different approach to this deformation problem. In particular, if the totally real 2 -sphere $P \subset Z$ is perturbed slightly, the family of disks holomorphic disks $\left(D^{2}, \partial D^{2}\right) \hookrightarrow(Z, P)$ will survive. In order to study large perturbations of $P$, however, one needs something like an energy estimate. Fortunately, the needed bound is freely available in the present context, as a consequence of the following observation:

Lemma 2. Let $\psi: \mathbb{C P}_{1} \rightarrow \mathbb{C P}_{1}$ be any orientation-reversing diffeomorphism, and let $P \subset \mathbb{C P}_{1} \times \mathbb{C P}_{1}$ be the graph of $\psi$. Then there is a Kähler metric $h$ on $Z=\mathbb{C P}_{1} \times \mathbb{C P}_{1}$ such that $P$ is Lagrangian with respect to the corresponding Kähler form $\omega$, and such that $\omega$ has de Rham cohomology class $[\omega]=2 \pi c_{1}(Z) \in H^{2}(Z, \mathbb{R})$.

Proof. Let $h_{2}$ be the standard metric on the unit 2-sphere, which we identify in the usual way with $\mathbb{C P}_{1}$. Then $h_{2}$ is Kähler, with Kähler form $\omega_{2}$. Set $\omega_{1}=-\psi^{*} \omega_{2}$. Since $\psi$ is an orientation-reversing diffeomorphism, $\omega_{1}$ is a positive 2 -form on $\mathbb{C P}_{1}$, and is therefore the area form of a unique conformal rescaling $h_{1}$ of $h_{2}$. Moreover, the total area of $\mathbb{C P}_{1}$ with respect to either $h_{1}$ or $h_{2}$ is $4 \pi$, so that $\left[\omega_{1}\right]$ and $\left[\omega_{2}\right]$ both represent $2 \pi c_{1}\left(\mathbb{C P}_{1}\right)$.

Now consider the product metric

$$
h=h_{1} \oplus h_{2}:=\varpi_{1}^{*} h_{1}+\varpi_{2}^{*} h_{2}
$$

where $\varpi_{j}: \mathbb{C P}_{1} \times \mathbb{C P}_{1} \rightarrow \mathbb{C P}_{1}, j=1,2$, are the factor projections. Then $h$ is a Kähler metric with Kähler form $\omega=\omega_{1} \oplus \omega_{2}:=\varpi_{1}^{*} \omega_{1}+\varpi_{2}^{*} \omega_{2}$. The restriction of $\omega$ to the graph of $P$ is then given by

$$
(\text { id } \times \psi)^{*}\left(\varpi_{1}^{*} \omega_{1}+\varpi_{2}^{*} \omega_{2}\right)=\omega_{1}+\psi^{*} \omega_{2}=\omega_{1}-\omega_{1}=0
$$

so $P$ is therefore Lagrangian. Finally,

$$
[\omega]=\varpi_{1}^{*}\left[\omega_{1}\right]+\varpi_{2}^{*}\left[\omega_{2}\right]=\varpi_{1}^{*} 2 \pi c_{1}\left(\mathbb{C P}_{1}\right)+\varpi_{2}^{*} 2 \pi c_{1}\left(\mathbb{C P}_{1}\right)=2 \pi c_{1}\left(\mathbb{C P}_{1} \times \mathbb{C P}_{1}\right),
$$

and the Kähler class $[\omega]$ is therefore independent of $\psi$, as claimed.

If $\psi: \mathbb{C P}_{1} \rightarrow \mathbb{C P}_{1}$ is an orientation-reversing diffeomorphism, it has degree -1 , and its graph $P$ therefore has homology class $(1,-1)$ in $H_{2}\left(\mathbb{C P}_{1} \times \mathbb{C P}_{1}, \mathbb{Z}\right) \cong \mathbb{Z} \oplus \mathbb{Z}$. The long exact sequence

$$
\cdots \rightarrow H_{2}(P) \rightarrow H_{2}(Z) \rightarrow H_{2}(Z, P) \rightarrow H_{1}(P) \rightarrow \cdots
$$

therefore tells us that $H_{2}\left(\mathbb{C P}_{1} \times \mathbb{C P}_{1}, P ; \mathbb{Z}\right) \cong \mathbb{Z}$, where the generator a can be realized as the image of either $\mathbb{C P}_{1} \times\{p t\}$ or $\{p t\} \times \mathbb{C P}_{1}$ in $\mathbb{C P}_{1} \times \mathbb{C P}_{1}$. As indicated by the de Sitter space example discussed above, the holomorphic disks $\left(D^{2}, \partial D^{2}\right) \rightarrow(Z, P)$ relevant to our problem are precisely those which belong to this generating class $\mathbf{a} \in H^{2}(Z, P)$. With respect to the Kähler metric $h$ of Lemma 2, these all have area $4 \pi$. 
Lemma 3. Let $\psi: \mathbb{C P}_{1} \rightarrow \mathbb{C P}_{1}$ be any orientation reversing diffeomorphism, let $P \subset \mathbb{C P}_{1} \times \mathbb{C P}_{1}$ be its graph, and let $F:(D, \partial D) \rightarrow(Z, P)$ be any holomorphic disk representing the generator

$$
\mathbf{a} \in H_{2}\left(\mathbb{C P}_{1} \times \mathbb{C P}_{1}, P ; \mathbb{Z}\right) \cong \mathbb{Z} .
$$

Then $F$ is a holomorphic embedding, is smooth up to the boundary, and sends the interior of $D$ to the complement of $P$. Moreover, $F(\partial D)$ is a smooth Jordan curve in $P \approx S^{2}$.

More generally, if $C$ is any holomorphic curve with boundary representing $\mathbf{a}$, then $C$ is non-singular, and is either the image of a holomorphic disk as described above, or is a factor $\mathbb{C P}_{1}$ of $\mathbb{C P}_{1} \times \mathbb{C P}_{1}$.

Proof. Consider the abstract oriented 2-sphere obtained by taking the double $D \cup \bar{D}$, where the two copies of the disk are identified along the boundary, $D$ is given the usual orientation coming from the unit disk in $\mathbb{C}$, and $\bar{D}$ is given the opposite orientation. Given $F$ as above, construct a continuous map $\hat{F}: D \cup \bar{D} \rightarrow \mathbb{C P}_{1}$ by

$$
\hat{F}(z)= \begin{cases}\varpi_{1} \circ F(z) & \text { if } z \in D \\ \psi^{-1} \circ \varpi_{2} \circ F(z) & \text { if } z \in \bar{D} .\end{cases}
$$

Since we have assumed that that $\psi$ is smooth, so is $P$, and the holomorphic map $F$ is therefore smooth up to the boundary [4]. Hence $\hat{F}$ is actually smooth when restricted to either $D$ or $\bar{D}$, and is moreover orientation-preserving at every regular point.

Now, equipping $\mathbb{C P}_{1}$ and $\mathbb{C P}_{1} \times \mathbb{C P}_{1}$ respectively with the Kähler forms $\omega_{1}$ and $\omega$ of the proof of Lemma 2, we have

$$
\int_{D \cup \bar{D}} \hat{F}^{*} \omega_{1}=\int_{D} F^{*}\left(\varpi_{1}^{*} \omega_{1}+\varpi_{2}^{*} \omega_{2}\right)=\int_{D} F^{*} \omega=4 \pi=\int_{\mathbb{C P}_{1}} \omega_{1}
$$

and it therefore follows that $\hat{F}$ has degree 1 . Moreover, since $\hat{F}$ is an orientationpreserving map at each of its regular points, the inverse image of any regular value must consist of exactly one point. We therefore conclude that $F(D)$ is the graph of a Riemann-mapping biholomorphism between two simply-connected domains with smooth boundary in $\mathbb{C P}_{1}$.

If $C$ is a singular Riemann surface in $Z$ with boundary on $P$, and if $[C]=\mathbf{a}$ in relative homology, we may first break $C$ up into its irreducible components, and then observe that, because each component must have area $\leq 4 \pi, C$ can in fact have only one irreducible component, with multiplicity 1 , and so must be irreducible. If $C$ has non-empty boundary, a straightforward generalization of the above doubling argument shows that $C \cup \bar{C}$ must be homeomorphic to $\mathbb{C P}_{1}$, and it therefore follows that $C$ is one of the disks we have already analyzed. On the other hand, if $C$ has no boundary, the fact that it must once again have area $4 \pi$ immediately implies that it must be a factor $\mathbb{C P}_{1}$.

If $P \subset \mathbb{C P}_{1} \times \mathbb{C P}_{1}$ is the graph of any orientation reversing diffeomorphism $\mathbb{C P}_{1} \rightarrow$ $\mathbb{C P}_{1}$, Gromov's compactness theorem [17, Theorem 4.6.1] therefore tells us that the space of all embedded holomorphic disks $(D, \partial D) \subset(Z, P)$ with $[(D, \partial D)]=\mathbf{a} \in$ $H_{2}(Z, P)$, together with the collection of lines of the form $\mathbb{C P}_{1} \times\{p t\}$ and $\{p t\} \times \mathbb{C P}_{1}$, forms a compact topological space. On the other hand, Lemma 3 also tells is than any 
such disk is diffeomorphically conjugate to one of the disks in our de Sitter example, and therefore has the same Maslov index. Since such a disk is therefore [13, 17] Fredholm regular, deformation theory implies that the space of such disks is a smooth 3-manifold - although, a priori, it might still be either empty or disconnected.

To show that the moduli space $M_{\psi}$ of disks is non-empty for each $\psi$, we first recall that the space of orientation-reversing diffeomorphisms of $S^{2}$ is connected, and that our deformation theory tells us that the set of $\psi$ for which disks $d o$ exist is open. It is therefore tempting to just try to invoke Gromov compactness to show that it is also closed. However, we know that sequences of disks can degenerate into factor $\mathbb{C P}_{1}$ 's, and we must control this degeneration in order to guarantee that we actually get a disk in the limit. We do so by introducing the function $\Omega: M_{\psi} \rightarrow(0,4 \pi)$ on the moduli space of disks which assigns to any disk $F:\left(D^{2}, \partial D^{2}\right) \rightarrow(Z, P)$ the number $\Omega(F)=\int_{D^{2}} F^{*} \varpi_{1}^{*} \omega_{2}$, where $\omega_{2}$ is the area form on the second factor. Since a sequence of disks $F_{j}$ can degenerate to $\mathbb{C P}_{1} \times\{p t\}$ only if $\Omega\left(F_{j}\right) \rightarrow 0$, and can degenerate to $\{p t\} \times \mathbb{C P}_{1}$ only if $\Omega\left(F_{j}\right) \rightarrow 4 \pi$, it follows that $\Omega$ is a proper map on the moduli space of disks. Taking limits as we vary $\psi$ thus shows that, for every $\psi$, the subset $\Omega^{-1}(t) \subset M_{\psi}$ is non-empty and compact for each $t \in(0,4 \pi)$; moreover, by Sard's theorem, this level set in any given $M_{\psi}$ is a smooth compact surface for almost every $t \in(0,4 \pi)$.

Given a choice of $\psi$, we now turn to the construction of an Einstein-Weyl structure on the moduli space $M=M_{\psi}$. Our first step in this direction is the construction of a conformal structure $[g]=[g]_{\psi}$ on $M$. To do this, we first observe that our deformation theory [13] allows us to identify the tangent space of $M$ at a given disk $(D, \partial D) \subset(Z, P)$ with the space of those holomorphic sections of the normal bundle $\left(\left.T Z\right|_{D}\right) / T D$ of $D \subset Z$ for which the boundary values are sections of of the normal bundle $\left(\left.T P\right|_{\partial D}\right) / T \partial D$ of $\partial D \subset P$. Because the Maslov index of the normal bundle of $D$ is 2 , this space is perfectly modeled by the space $\mathfrak{s l}(2, \mathbb{R})$ of infinitesimal Möbius transformations of the disk. Consequently, up to rescalings, each tangent space of $M$ carries a natural Lorentz metric modeled on the Killing form on $\mathfrak{s l}(2, \mathbb{R})$. The trichotomy of vectors into space-like, null, and time-like thus corresponds to the classification of infinitesimal Möbius transformations of the disk as hyperbolic, parabolic, or elliptic. A space-like vector on $M$ therefore corresponds to an infinitesimal variation of the corresponding disk with two distinct zeroes on $\partial D$; a null vector corresponds to an infinitesimal variation with a repeated zero on $\partial D$; and a time-like vector corresponds to an infinitesimal variation with a single zero in the interior of $D$, but none along $\partial D$.

In particular, a one-parameter family of disks is time-like with respect to this conformal structure $[g]$ iff the associated variation normal vector fields have no zeroes along the boundaries. By Lemma 3, this in particular means that the corresponding Jordan curves are nested, so as not to overlap, moving inward or outward at a non-zero rate everywhere. Projecting these disks into $\mathbb{C P}_{1}$ by $\varpi_{2}$, we thus see that the function $\Omega: M \rightarrow(0,4 \pi)$, which measures the area of these projections, has non-vanishing derivative along any time-like curve. Consequently, $M$ carries a time orientation for which $\Omega$ is increasing along all future-pointing time-like curves. More strikingly, the proper function $\Omega: M \rightarrow(0,4 \pi)$ therefore has no critical points, and integration of a time-like vector field $v$ with $v \Omega=1$ thus gives us a diffeomorphism $M \approx \Sigma \times(0,4 \pi)$, 
where $\Sigma$ is the smooth compact surface $\Omega^{-1}(0)$, and where $\Omega$ has now become factor projection to the second factor $(0,4 \pi)$ of the product. Since the compactness of $\Sigma$ guarantees that any endless time-like or null curve in $M$ must have $\Omega \searrow 0$ at one extremity and $\Omega \nearrow 4 \pi$ at the other, the intermediate value theorem forces the level set $\Sigma \times\{0\}$ to meet every such curve. Thus $(M,[g])$ has a Cauchy surface, and is therefore globally hyperbolic.

Now, following any past-endless time-like curve backwards in $M$ corresponds to a nested family of disks in the second-factor $\mathbb{C P}_{1}$ with unique point as intersection, since we know that the Gromov limit of the corresponding disks in $Z$ is one of the lines $\mathbb{C P}_{1} \times\{p t\}$. This gives us a unique limit point on $\mathbb{C P}_{1}$. Following the time-like factors of $M \approx \Sigma \times(0,4 \pi)$ backwards therefore gives us homeomorphism from $\Sigma$ to $\mathbb{C P}_{1}$, and in particular giving $\Sigma \approx S^{2}$ a preferred orientation. Thus $M \approx S^{2} \times \mathbb{R}$ is space-time oriented, as promised.

We now endow $M$ with a Weyl connection $\nabla$ compatible with $[g]$. To do so, we begin by describing its geodesics. The time-like geodesics are the family of holomorphic disks through a given point $x \in Z-P$. The null geodesics are the families of disks passing through a given point $x \in P$ with specified tangent. (These really are null geodesics of $[g]$, because [20] they belong to the boundary of the future of a point, without actually entering its future.) Finally, the space-like geodesics are the families of disks passing though a pair of distinct points $x \neq y$ of $P$. Of course, is not immediately obvious that there really is a connection which has these curves as its geodesics, but Fuminori Nakata has written out a careful proof [18, Proposition 8.1] of this crucial fact by methods based on our study of Zoll surfaces [14, Theorem 4.7]. Requiring the connection $\nabla$ also be $[g]$-compatible then specifies it completely. The fact that this connection is Einstein-Weyl then follows [8] from the fact that every null geodesic belongs to a totally geodesic null hypersurface - namely, the family of disks passing through a given point of $P$.

However, we prefer a second method of proving the existence of $([g], \nabla)$, as this will turn out to be better adapted to analyzing the geometry at infinity. First notice that each of our disks $D$ has a canonical lift to the projectivized tangent bundle $\mathbb{P} T Z=\mathbb{P} T\left(\mathbb{C P}_{1} \times \mathbb{C P}_{1}\right)$, given by its tangent bundle $T D \subset T Z$; moreover, this lift is automatically Legendrian with respect to the tautological contact structure on $\mathbb{P} T Z=\mathbb{P} T^{*} Z$. Moreover, since each disk $D$ is the graph of a biholomorphism between simply connected domains in $\mathbb{C P}_{1}$, its tangent line $T D$ is never vertical or horizontal, and therefore may be viewed as a non-zero element of the line bundle $\mathcal{O}(-2,2)$ of linear maps between the tangent space of the first and second factors. However, the complement of the zero section in $\mathcal{O}(-1,1)$ is the universal cover of the complement of the zero section in $\mathcal{O}(-2,2)$. Moreover, the complement of the zero section in $\mathcal{O}(-1,1)$ is exact $\mathbb{C P}_{3}$ minus two skew lines $L_{1}$ and $L_{2}$, the projection to $\mathbb{C P}_{1} \times \mathbb{C P}_{1}$ being given by $\left[z^{1}: z^{2}: z^{3}: z^{4}\right] \mapsto\left(\left[z^{1}: z^{2}\right],\left[z^{3}: z^{4}\right]\right)$ if we choose our coordinates so that $L_{1}$ and $L_{2}$ are given by $z^{1}=z^{2}=0$ and $z^{3}=z^{4}=0$, respectively. We thus obtain two preferred liftings of each holomorphic disk $D$ to a disk in $\mathbb{C P}_{3}$. Now the inverse image of $P \subset \mathbb{C P}_{1} \times \mathbb{C P}_{1}$ contains a double cover of the projectivized tangent bundle $\mathbb{R} P T P$, and this cover is necessarily diffeomorphic to the unit tangent bundle $U T P$ of $P$, in a manner that is completely canonical up to an overall choice of sign. Making such a choice of sign once and for all, we then get a unique lift $\tilde{D}$ of each 
disk $D$ by requiring that $\partial \tilde{D} \subset U T P$ be the natural lift of the oriented Jordan curve curve $\partial D \subset P$. Each such disk $\tilde{D} \subset \mathbb{C P}_{3}$ then has its boundary on the totally real submanifold $U T P \approx \mathbb{R P}^{3}$, and is Legendrian with respect to the complex contact form $\theta=z^{1} d z^{2}-z^{2} d z^{1}+z^{3} d z^{4}-z^{4} d z^{3}$.

Each of these disks $\tilde{D}$ represents the generator of $H_{2}\left(\mathbb{C P}_{3}, U T P\right) \cong \mathbb{Z}$, so the contact line bundle becomes $\mathcal{O}(2)$ on the abstract double $\tilde{D} \cup \tilde{D}$ of any such disk. Because $\tilde{D}$ is Legendrian, the normal bundle of its double is therefore $J^{1} \mathcal{O}(2) \cong$ $\mathcal{O}(1) \oplus \mathcal{O}(1)$. Moving these disks by the $S^{1}$ action $\left[z^{1}: z^{2}: z^{3}: z^{4}\right] \mapsto\left[z^{1}: z^{2}: e^{i \theta} z^{3}:\right.$ $\left.e^{i \theta} z^{4}\right]$, we thus obtain a 4 -parameter family $M \times S^{1}$ of disks in $\left(\mathbb{C P}_{3}, U T P\right)$ with doubled normal bundle $\mathcal{O}(1) \oplus \mathcal{O}(1)$. It therefore follows [15, Prop. 10.1] that $M \times S^{1}$ carries an $S^{1}$-invariant self-dual conformal structure of signature $(++--)$, and the quotient of this geometry by $S^{1}$ therefore $[2,10]$ gives us the promised Lorentzian Einstein-Weyl structure on $M$.

Now orbits of the the $S^{1}$-action on $U T P \subset \mathbb{C P}_{3}$ are the fibers of the projection $U T P \rightarrow P$, and each of these circles is thus the intersection of a fiber of $\mathcal{O}(-1,1) \rightarrow Z$ with $U T P$. Each of these circles is therefore contained in a unique projective line $L$ in $\mathbb{C P}_{3}$ which meets both of the skew lines $L_{1}$ and $L_{2}$. Such a circle then divides $L$ into two disks, one of which meets only $L_{1}$, while the other meets only $L_{2}$. We thus obtain two $S^{2}$-families of essentially explicit holomorphic disks $(\Delta, \partial \Delta) \subset\left(\mathbb{C P}_{3}, U T P\right)$. For any such disk $\Delta$, the abstract double $\Delta \cup \bar{\Delta}$ is concretely realized as $L \subset \mathbb{C P}_{3}$, and the double therefore has abstract normal bundle $\mathcal{O}(1) \oplus \mathcal{O}(1)$. Deformation theory $[6,13]$ therefore gives us a 4 -parameter family of disks in $\left(\mathbb{C P}_{3}, U T P\right)$ near any such $\Delta$. However, the infinitesimal variations of such a disk which continue to meet the relevant line $L_{1}$ or $L_{2}$ form a linear subspace of codimension 2 . It follows that, aside from our initial 2-spheres of essentially explicit disks, all the other disks $\Delta^{\prime}$ of this family are disjoint from $L_{1}$ and $L_{2}$, and so are contained in $\mathcal{O}(-1,1)$ minus the zero section. Projecting any such disk $\Delta^{\prime}$ into $\mathbb{C P}_{1} \times \mathbb{C P}_{1}$ then gives us a holomorphic disk with boundary on $P$. However, since the natural homomorphisms $H_{2}\left(\mathbb{C P}_{3}-\left(L_{1} \cup L_{2}\right), U T P\right) \rightarrow H_{2}\left(\mathbb{C P}_{3}, U T P\right)$ and $H_{2}\left(\mathbb{C P}_{3}-\left(L_{1} \cup L_{2}\right), U T P\right) \rightarrow$ $H_{2}\left(\mathbb{C P}_{1} \times \mathbb{C P}_{1}, P\right)$ are both isomorphisms, the images of any of these disks represents the generator of $H_{2}(Z, P)$. By Lemma 3 , the projection of any such $\Delta^{\prime}$ is therefore a disk $D$ of our original family. However, thinking of $\Delta^{\prime}$ as a section of $\left.\mathcal{O}(-1,1)\right|_{D}$, we now see that it must be obtained from the canonical lift $\tilde{D}$ by multiplication by a holomorphic function on $D$ with unit modulus on $\partial D$; and since any such function is constant, it follows that each $\Delta^{\prime}$ actually belongs to our previous family $M \times S^{1}$. It follows that $M \times S^{1}$ can be compactified by adding the two $S^{2}$-families of disks $\Delta$, and the result is now an $\left(S^{2} \times S^{2}\right)$-family of disks in $\left(\mathbb{C P}_{3}, U T P\right)$ with doubled normal bundle $\mathcal{O}(1) \oplus \mathcal{O}(1)$. The moduli space $Y \approx S^{2} \times S^{2}$ therefore [15] carries a splitsignature self-dual metric which is invariant under an $S^{1}$ action which just rotates one $S^{2}$ factor about an axis, while acting trivially on the other. The quotient $X=$ $Y / S^{1}$ therefore [12] carries an induced conformal structure, and its interior $M$ carries an Einstein-Weyl structure whose singularity at $\partial X$ is exactly as described in our definition of conformal compactness. Thus any orientation-reversing diffeomorphism $\psi: \mathbb{C P}_{1} \rightarrow \mathbb{C P}_{1}$ determines a globally hyperbolic, conformally compact, Lorentzian Einstein-Weyl manifold, exactly as claimed. 
Of course, it remains to check that our two constructions really are inverses of one another. In passing from the twistor-disk construction to the associated scattering map, this is relatively straightforward, since in the twistor picture a null geodesic is just the family of disks with boundaries tangent to a fixed element of UTP. The opposite direction is rather more subtle, but just comes down to the fact that the mini-twistor space of a Lorentzian Einstein-Weyl space can be constructed, in analogy with [9] and [14], by starting with the space of future-pointing time-like geodesics, equipped with the complex structure obtained by $90^{\circ}$ rotation of Jacobi fields, and then adjoining a quotient of the space of null geodesics that represents the space of totally geodesic null surfaces. Details are left to the interested reader.

We conclude by mentioning several surprising features of the present correspondence that might offer fruitful directions for further investigation. First, the availability of a Kähler metric for which $P$ is Lagrangian allowed us to prove results here which are markedly sharper than those currently known for our earlier twistor-disk constructions $[14,15]$, and we therefore wonder if some of our previous results could be sharpened by means of related techniques. Second, we find it interesting that, $a$ posteriori, any divergent sequence of lifted disks $\tilde{D}_{j} \subset \mathbb{P} T Z$ must subconverge to a singular curve consisting of the Legendrian lift of a $\mathbb{C P}_{1}$ and half of a fiber of $\mathbb{P} T Z \rightarrow Z$; and we wonder if this might instead be shown more directly by some Gromov-type compactness argument. Third, the Legendrian nature of the lifted disks $\tilde{D}$ means that the associated embedding $M \hookrightarrow Y$ is actually umbilic, and that [11] the complement of a suitable hypersurface in $Y$ must therefore carry a self-dual Einstein metric that one would like to understand more explicitly. Finally, as was brought to our attention by Nakata's independent investigation [18], the Einstein-Weyl structures constructed here are all actually space-like Zoll, in the sense that their space-like geodesics are all simple closed curves, and we are intrigued by Nakata's very natural problem of classifying all possibles Einstein-Weyl 3-manifolds with this property — are they all given by the present construction? But we will just leave these unresolved issues for the interested reader to ponder, and simply hope that some of them may eventually lead to interesting new avenues of research.

\section{References}

[1] N. Berkovits and E. Witten, Conformal supergravity in twistor-string theory, J. High Energy Phys. (2004), no. 8, 009, 36 pp. (electronic).

[2] D. M. J. Calderbank and H. Pedersen, Einstein-Weyl geometry, in Surveys in Differential Geometry: Essays on Einstein Manifolds, Surv. Differ. Geom., VI, 387-423, Int. Press, Boston, MA (1999).

[3] É. Cartan, Sur une classe d'espaces de Weyl, Ann. Sci. École Norm. Sup. (3) 60 (1943) 1-16.

[4] E. M. Chirka, Regularity of the boundaries of analytic sets, Mat. Sb. (N.S.) 117(159) (1982), no. 3, 291-336, 431.

[5] R. Geroch, Domain of dependence, J. Mathematical Phys. 11 (1970) 437-449.

[6] J. Globevnik, Perturbation by analytic discs along maximal real submanifolds of $\mathbf{C}^{N}$, Math. Z. 217 (1994), no. 2, 287-316.

[7] C. R. Graham and J. M. Lee, Einstein metrics with prescribed conformal infinity on the ball, Adv. Math. 87 (1991), no. 2, 186-225.

[8] N. J. Hitchin, Complex manifolds and Einstein's equations, in Twistor Geometry and Nonlinear Systems (Primorsko, 1980), 73-99, Springer (1982).

[9] —, Monopoles and geodesics, Comm. Math. Phys. 83 (1982), no. 4, 579-602. 
[10] P. E. Jones and K. P. Tod, Minitwistor spaces and Einstein-Weyl spaces, Classical Quantum Gravity 2 (1985), no. 4, 565-577.

[11] C. LeBrun, $\mathcal{H}$-space with a cosmological constant, Proc. Roy. Soc. London Ser. A 380 (1982), no. $1778,171-185$.

[12] — Self-dual manifolds and hyperbolic geometry, in Einstein metrics and Yang-Mills connections (Sanda, 1990), Vol. 145 of Lecture Notes in Pure and Appl. Math., 99-131, Dekker, New York (1993).

[13] — Twistors, holomorphic disks, and Riemann surfaces with boundary, in Perspectives in Riemannian geometry, Vol. 40 of CRM Proc. Lecture Notes, 209-221, Amer. Math. Soc., Providence, RI (2006).

[14] C. LeBrun and L. J. Mason, Zoll manifolds and complex surfaces, J. Differential Geom. 61 (2002), no. 3, 453-535.

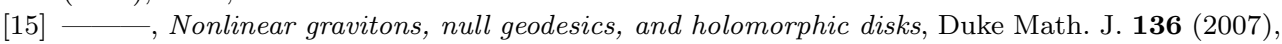
no. 2, 205-273.

[16] L. J. Mason, Global anti-self-dual Yang-Mills fields in split signature and their scattering, J. Reine Angew. Math. 597 (2006) 105-133.

[17] D. McDuff and D. Salamon, $J$-holomorphic Curves and Symplectic Topology, American Mathematical Society, Providence, RI (2004), ISBN 0-8218-3485-1.

[18] F. Nakata, A construction of Einstein-Weyl spaces via LeBrun-Mason type twistor correspondence (2008). E-print arXiv:0806.2696 [math.DG].

[19] R. Penrose, Structure of space-time, in Battelle rencontres. 1967 Lectures in Mathematics and Physics, Edited by Cecile M. DeWitt and John A. Wheeler, 121-235, W. A. Benjamin, Inc., New York-Amsterdam (1968).

[20] — Techniques of Differential Topology in Relativity, Society for Industrial and Applied Mathematics, Philadelphia, Pa. (1972). Conference Board of the Mathematical Sciences Regional Conference Series in Applied Mathematics, No. 7.

[21] E. Witten, Perturbative gauge theory as a string theory in twistor space, Comm. Math. Phys. 252 (2004), no. 1-3, 189-258

Department of Mathematics, SUny Stony Brook, Stony Brook, Ny 11794-3651, USA

E-mail address: claude@math.sunysb.edu

Mathematical Institute, University of Oxford, 24-29 St. Giles, Oxford OX1 3LB, UK

E-mail address: Imason@maths.ox.ac.uk 